\title{
Sarcoma fibromixoide de bajo grado como contenido de hernia perineal. Reporte de un caso
}

\author{
Low-grade fibromyxoid sarcoma as a content of perineal hernia. A case report
}

Danna Auria-Aspiazu*, Omar Parrales-Calderón² y Fernando Moncayo-Asnalema ${ }^{3}$

${ }^{1}$ Servicio de Cirugía General, Instituto Oncológico Nacional Dr. Juan Tanca Marengo, ION SOLCA; ${ }^{2}$ Servicio de Coloproctología, Hospital de Especialidades Dr. Abel Gilbert Pontón; ${ }^{3}$ Servicio de Cirugía General, Hospital de Especialidades Dr. Abel Gilbert Pontón. Guayaquil, Ecuador

\begin{abstract}
Resumen
Antecedentes: El sarcoma fibromixoide de bajo grado es una neoplasia rara de histología someramente benigna, pero con un comportamiento agresivo metastásico tardío. Las hernias perineales son de presentación inusitada y no existen más de 100 casos reportados en la literatura mundial. Discusión: La presentación de esta neoplasia fibroblástica es poco frecuente, y aún más como contenido de una hernia perineal primaria. Conclusión: Es una neoplasia infrecuente que se presenta en aproximadamente el $0.7 \%$ de los casos, con comportamiento agresivo metastásico y con necesidad de tratamiento adyuvante.
\end{abstract}

Palabras clave: Hernia perineal. Sarcoma fibromixoide. Sarcoma fibromixoide de bajo grado. Tejidos blandos.

\begin{abstract}
Background: Low-grade fibromyxoid sarcoma is a rare neoplasm with apparently benign histology but with late aggressive metastatic behavior. The perineal hernias are unusual, there are no more than 100 cases reported in the world literature. Discussion: The presentation of this fibroblastic neoplasm is rare and makes it even more rare if it is as the content of a primary perineal hernia. Conclusion: It is an infrequent neoplasm that occurs in approximately $0.7 \%$ of all cases, with aggressive metastatic behavior and in need of adjuvant treatment.
\end{abstract}

Key words: Perineal hernia. Fibromyxoid sarcoma. Low-grade fibromyxoid sarcoma. Soft tissues.

\section{Introducción}

El sarcoma fibromixoide es una neoplasia rara que supone aproximadamente el $0.7 \%$ de los tumores de partes blandas. Descrito por primera vez por Evans en 1987, es un tumor de origen fibroblastico de características clínicas e histológicas en apariencia benignas, pero presenta un comportamiento local y metastásico muy agresivo. Su localización más habitual es en las extremidades proximales, sobre todo en los muslos, y luego en la pared torácica de adultos jóvenes entre los 25 y 46 años, con una edad promedio de 35 años, y con mayor predominio en el sexo masculino. Se han informado no más de 150 casos en todo el mundo por ser una afección poco conocida hasta el momento ${ }^{1,2}$.

Debido a la baja incidencia de este tipo de tumores y a su forma de presentación tan inusual como contenido herniario, se describe este caso con el fin de 
realizar una revisión de la literatura internacional, tanto de su epidemiología como de las características histopatológicas, la presentación clínica y las opciones terapeúticas que existen hasta el momento.

El objetivo de este artículo es documentar un caso de sarcoma de variedad fibromixoide de bajo grado como contenido de una hernia perineal posterior primaria en una mujer joven. Se describen a continuación la incidencia, las características clínicas e histopatológicas, su presentación inusual y el manejo terapéutico de este tipo de neoplasia, por ser muy infrecuente de acuerdo con la verificación de la literatura disponible.

\section{Caso clínico}

Mujer de 31 años con antecedentes de asma bronquial y antecedentes gineco-obstétricos de cuatro gestas (dos abortos y dos cesáreas), que acude a la emergencia por presentar un cuadro clínico de 5 meses de evolución posterior a un trauma glúteo por caída desde su propia altura, que presenta desde hace unas semanas edema, rubor, calor y dolor en la región vulvar izquierda. Se realiza ecografía de partes blandas, la cual reporta una glándula de Bartholini aumentada de tamaño, presencia de fístula que desciende del labio al glúteo izquierdo de $1.72 \times 0.59 \mathrm{~cm}$, y una imagen enquistada de bordes irregulares heterogénea de $3.39 \times 3.16 \mathrm{~cm}$ con un volumen de $10 \mathrm{~cm}^{3}$ e imagen interna anecoica heterogénea en el glúteo izquierdo. La paciente es manejada de forma ambulatoria con analgésicos y antibioticoterapia (ibuprofeno, $400 \mathrm{mg} / 8 \mathrm{~h}$ por vía oral, y azitromicina, $500 \mathrm{mg} / 12 \mathrm{~h}$ por 3 días), y seguimiento por consulta externa.

Tres días más tarde la paciente se presenta en la consulta externa con una colección hematopurulenta en la pared lateral izquierda de la vagina, el periné izquierdo y la región glútea izquierda, que al examen vaginal produce salida y descompresión de la masa de aspecto quístico. Se realiza ecografía pélvica, la cual evidencia una imagen hipoecogénica que mide $15 \times 15 \times 16 \mathrm{~cm}$, con un volumen aproximado de $1200 \mathrm{ml}$, en relación con una colección aparentemente retroperitoneal que se dirige a la izquierda y desplaza el útero hacia la derecha, que mide $69 \times 33 \mathrm{~mm}$; endometrio ecogénico fino sin colecciones en su interior y ovarios no valorables.

Se decide manejo integral en conjunto con el servicio de cirugía general, que indica la realización de una resonancia magnética abdominopélvica en la que se evidencia una lesión expansiva tumoral en la región pélvica que se extiende a nivel cefálico a la altura de L4 supraumbilical, que comprime y desplaza hacia la izquierda el cuerpo uterino, se introduce a través de

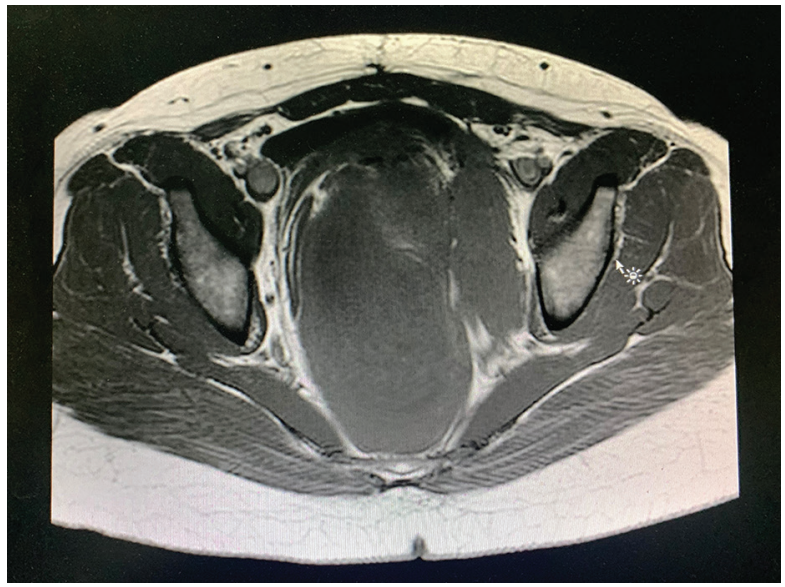

Figura 1. Corte axial de resonancia magnética abdominopélvica en el que se evidencia una lesión tumoral expansiva de intensidad sólida, de bordes lobulados, hipointensa en secuencias T1.

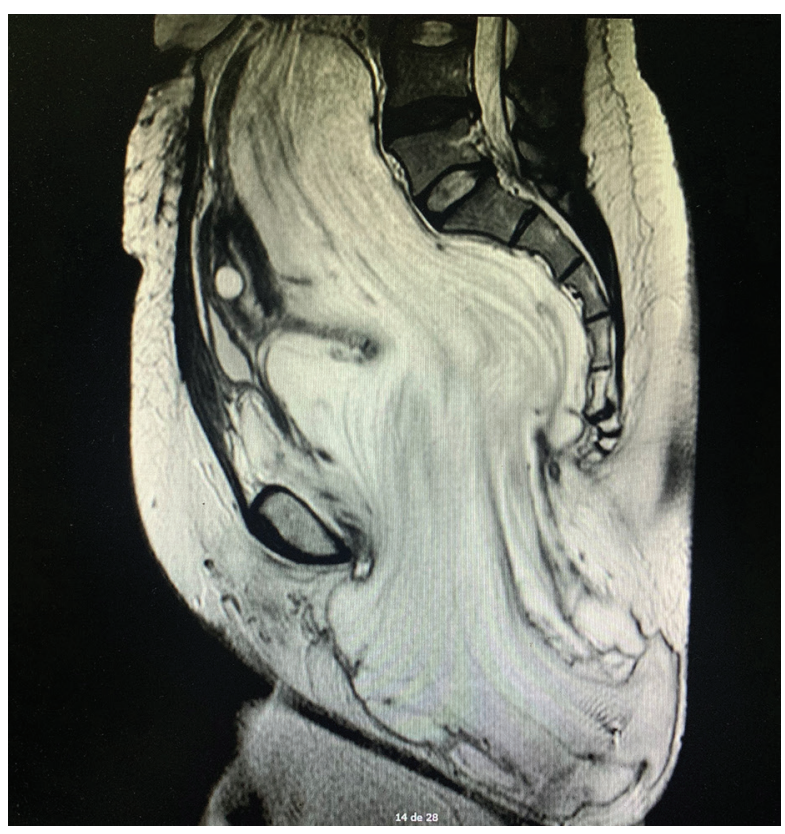

Figura 2. Corte sagital de resonancia magnética abdominopélvica que muestra una lesión hiperintensa heterogénea en secuencias T2 con captación heterogénea del contraste paramagnético, localizada en la región pélvica.

la pelvis menor y desplaza el recto sigmoides, y se desliza a través del espacio isquiopúbico izquierdo hacia la región perineal y la región glútea paramediana izquierda, que mide $34 \mathrm{~cm}$, por un eje anteroposterior de mayor extensión a nivel glúteo de $13 \mathrm{~cm}$ y un eje transverso en la cavidad pélvica de $10 \mathrm{~cm}$, ocupando el espacio presacro y prevertebral, y que comprime y desplaza la vejiga (Figs. 1 y 2). Se decide realizar exámenes de laboratorio e interconsultas a 


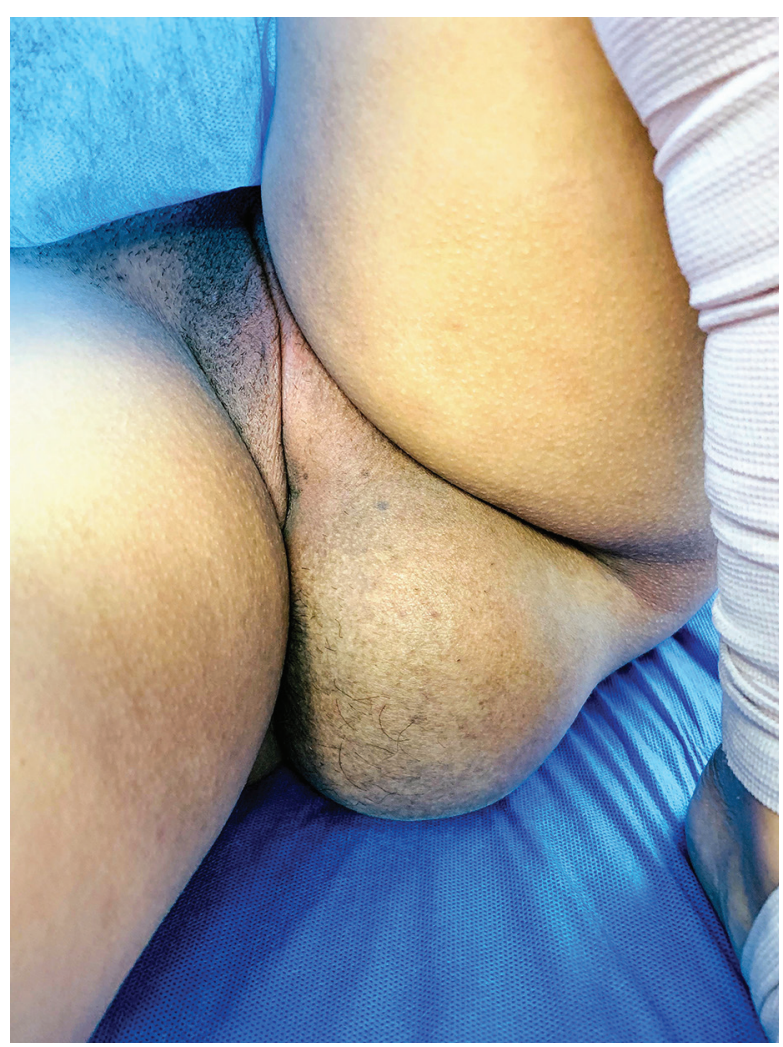

Figura 3. Lesión tumoral herniaria localizada en la región perineal y la región glútea paramediana izquierda.

anestesiología y cardiología para realizar una exploración abdominopélvica (Fig. 3).

Se realiza laparotomía exploradora y dentro de los hallazgos se evidencia una gran masa de aspecto quístico gelatinosa que desplaza el útero, la vejiga y el uréter hacia la derecha, y que envuelve al uréter izquierdo en su trayecto distal, con debilidad de estructuras musculares del piso pélvico. No se logra obtener un plano de clivaje entre el saco herniario que protruye hacia la región perineal posterior; sin embargo, se procede a la disección y separación de la masa de las estructuras pélvicas para su exéresis. Con un sangrado aproximado de $900 \mathrm{ml}$ y una transfusión transquirúrgica de dos paquetes de glóbulos rojos concentrados, se procede al cierre de la cavidad abdominal previa colocación de un drenaje tipo Hemovac por contraapertura dirigido al fondo de saco. Se envía la tumoración al servicio de patología para su estudio.

La paciente cursa el posquirúrgico con antibioticoterapia, con leve hematuria transitoria que cede a las 24 horas y drenaje de líquido serohemático de aproximadamente $30-45 \mathrm{ml}$ diarios. Se da el alta a los 5 días de la intervención.

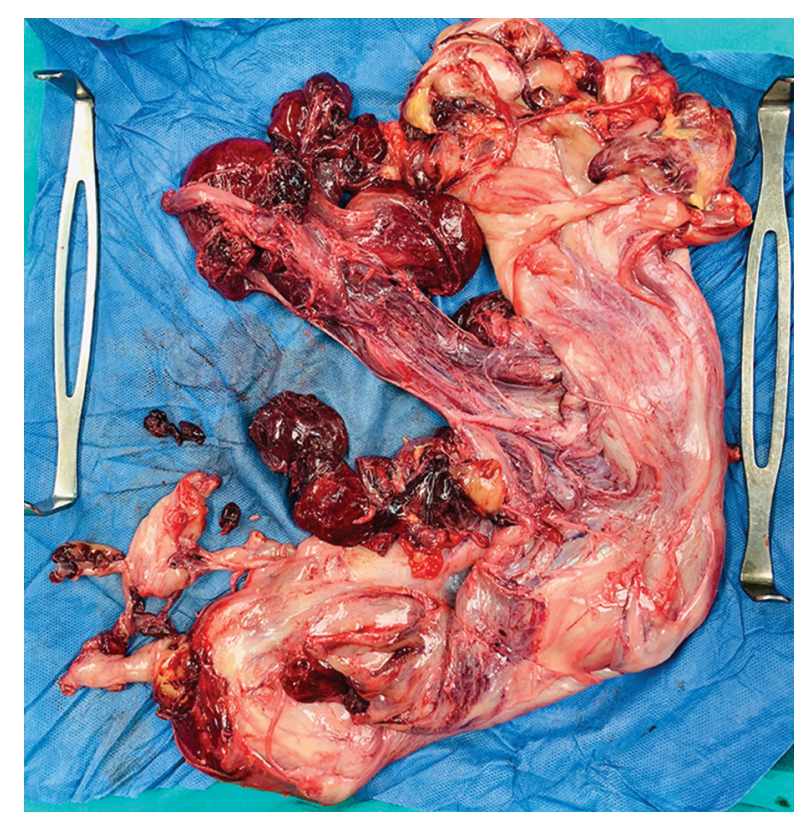

Figura 4. Lesión tumoral que mide $30 \times 22 \times 9 \mathrm{~cm}$, de consistencia elástica, superficie irregular, consistencia elástica, color pardo amarillento y aspecto mixoide.

La descripción macroscópica histopatológica reporta fragmentos de tejido que miden $30 \times 22 \times 9 \mathrm{~cm}$, de consistencia elástica, que en conjunto pesan $1300 \mathrm{~g}$, de forma y superficie irregulares, de color blanco grisáceo con áreas violáceas. Al corte se aprecia una consistencia elástica, heterogénea, de color pardo amarillenta y de aspecto mixoide, concordante con sarcoma fibromixoide de bajo grado (Fig. 4). El estudio inmunohistoquímico reporta positividad para vimentina y desmina, y negatividad para $S 100$, actina de músculo liso 1A4, músculo estriado AC, CD117, CD99, CD34, antígeno de membrana epitelial y queratina de amplio espectro, lo que confirma el diagnóstico.

La paciente está recibiendo tratamiento adyuvante con quimioterapia y radioterapia.

\section{Discusión}

Los tumores de partes blandas constituyen un grupo heterogéneo de tumores, tanto por su presentación histológica como por la diversidad de su inmunohistoquímica. Por lo general derivan de las células mesenquimales primitivas multipotenciales que pueden generar varias líneas celulares mientras se produce su transformación neoplásica. El sarcoma fibromixoide de bajo grado supone aproximadamente el $0.7 \%$ de los tumores de partes blandas. Descrito por primera vez por Evans en 1987, es de origen fibroblástico y posee 
características clínicas e histológicas aparentemente benignas, pero presenta un comportamiento local y metastásico muy agresivo, con una tasa alta de recurrencia local aproximada del $33 \%$ y de metástasis del $58 \%$. Su incidencia es incierta ${ }^{1,2}$. Hasta el momento existen 26 series de casos en la literatura en lengua inglesa, y en 15 de ellas se utilizan tecnicas moleculares para su diagnostico ${ }^{3}$.

Muestra predominio por el sexo masculino y los adultos jóvenes entre 25 y 46 años. Se localiza con mayor frecuencia en las extremidades inferiores, principalmente en el muslo, aunque también puede aparecer en la pared torácica, la axila, el hombro, el glúteo, la región inguinal o el cuello. Se han descrito unos pocos casos en el retroperitoneo, el mesenterio de intestino delgado, la pelvis, el mediastino y el ligamento falciforme. La mayoría se localizan de forma subfascial; rara vez tiene localización subcutánea o dérmica ${ }^{2,4,5}$.

Se presenta como un tumor de tejidos blandos de crecimiento lento y poco doloroso. En ocasiones puede cursar con hemorragia tumoral y es infrecuente que origine síntomas neurológicos o vasculares por compresion o estiramiento de nervios o de bursas adyacentes, dependiendo de la localización anatómica ${ }^{4,6}$.

El diagnóstico diferencial es amplio e incluye variantes mixoides del neurofibroma, leiomioma, hemangiopericitoma, fibromatosis desmoide, tumor maligno de nervio periférico, liposarcoma de células fusiformes y angiomixoma ${ }^{1,7}$.

En el estudio histológico suelen observarse fibroblastos, con áreas mixoides, con un patrón de distribución en remolino, con mínimo pleomorfismo nuclear, citoplasma eosinófilo claro y escasa o nula actividad mitótica. En cuanto a la inmunohistoquímica, la mayoría muestran positividad a vimentina y negatividad para queratina, desmina, actina, proteína S-100, CD34, CD31 y antígeno epitelial de membrana ${ }^{5,6}$.

El análisis molecular reacción en cadena de la polimerasa con transcriptasa inversa (RT-PCR) y la hibridación fluorescente in situ (FISH) que detecta la transcripción quimérica del gen de fusión FUS-CREB3L2 y el reordenamiento del gen FUS confirma y apoya los resultados morfológicos e inmunohistoquímicos ${ }^{8}$.

El tratamiento de elección es la cirugía, aunque la resección por lo general es incompleta ya que son tumores circunscritos pero no encapsulados. La quimioterapia y la radioterapia no han demostrado modificar el curso de la enfermedad ni mejorar la supervivencia. Las metástasis diseminadas pueden ser manejadas con tratamiento paliativo. No existen guías específicas para la enfermedad metastásica y recurrente, pues el abordaje depende del estado del paciente y del resultado histopatológico ${ }^{1,4}$.

\section{Conclusión}

El sarcoma fibromixoide de bajo grado es un tumor infrecuente y puede simular otras neoplasias de partes blandas. Se describen altas tasas de recidivas y metástasis. El diagnóstico final requiere estudio histopatológico, con realización de inmunohistoquímica y correlación clínica.

\section{Responsabilidades éticas}

Protección de personas y animales. Los autores declaran que para esta investigación no se han realizado experimentos en seres humanos ni en animales.

Confidencialidad de los datos. Los autores declaran que han seguido los protocolos de su centro de trabajo sobre la publicación de datos de pacientes.

Derecho a la privacidad y consentimiento informado. Los autores han obtenido el consentimiento informado de los pacientes y/o sujetos referidos en el artículo. Este documento obra en poder del autor de correspondencia.

\section{Financiamiento}

Los autores declaran que no se obtuvo financiamiento de ninguna organización o institución para la realización de este artículo.

\section{Conflicto de intereses}

Los autores declaran que no existen conflictos de intereses.

\section{Bibliografía}

1. Tinoco-Tellez L, Aguirre Trigueros J, Dominguez-Gasca L, Dominguez-Carrillo L. Sarcoma fibromixoide de bajo grado. Acta Médica. Grupo Ángeles. 2016;14:230-4.

2. Cruz-Benítez L, Cortés-Cárdenas S, Farías-Alarcón M, Tenorio-Torres J, Ramírez-Heredia J, Conde-Vazquez E. Sarcoma fibromixoide de región sacra. Reporte de caso. GAMO. 2011;10:319-25.

3. Mengtian L, Huijiao C, Dengchao S, Min C, Zhang Z, Hongying Z. Low-grade fibromyxoid sarcoma: a clinicopathologic and molecular study of 10 genetically confirmed cases. Int J Clin Exp Pathol. 2018;11:5860-8.

4. Solano Iturri G, Blanco Sampascual S, Orive Cura V. Tumor fibromixoide de bajo grado. Gastroenterol Hepatol. 2011;34:56-7.

5. Jain S, Xu R, Prieto VG, Lee P. Molecular classification of soft tissue sarcomas and its clinical applications. Int J Clin Exp Pathol. 2010;3:416-28.

6. Scoggins CR, Pisters PW. Diagnosis and management of soft tissue sarcomas. Adv Surg. 2008;42:219-28.

7. Alfaro-Cervelló C, Benavent Casanova O, Nieto G, Mares Diago FJ, Navarro S. Sarcoma fibromixoide de bajo grado, un diagnóstico diferencial esencial en los tumores mixoides de apariencia benigna. Rev Esp Patol. 2018;51:178-82.

8. Sambri A, Righi A, Tuzzato G, Donati D, Bianchi G. Low-grade fibromyxoid sarcoma of the extremities: a clinicopathologic study of 24 cases and review of the literature. Pol P Pathol. 2018;69:219-25. 